\title{
Autocontinuity from below of Set Functions and Convergence in Measure
}

Jun Li, Masami Yasuda, and Ling Zhou

\begin{abstract}
In this note, the concepts of strong autocontinuity from below and strong converse autocontinuity from below of set function are introduced. By using four types of autocontinuity from below of monotone measure, the relationship between convergence in measure and pseudo-convergence in measure for sequence of measurable function are discussed.
\end{abstract}

Keywords: Monotone measure, Autocontinuity from below, Convergence in measure, Pseudo-convergence in measure.

\section{Introduction}

In non-additive measure theory, there are several different kinds of convergence for sequence of measurable functions, such as almost everywhere convergence, pseudo-almost everywhere convergence, convergence in measure, and convergence pseudo-in measure. The implication relationship between such convergence concepts are closely related to the structural characteristics of set functions. In this direction there are a lot of results ( $[5,7,2,6,6,3,10,4,8,9,11,12,14,15])$.

Jun Li

School of Science, Communication University of China, Beijing 100024, China e-mail: lijun@cuc.edu.cn

Masami Yasuda

Department of Mathematics \& Informatics, Faculty of Science, Chiba University,

Chiba 263-8522, Japan

e-mail: yasuda@math.s.chiba-u.ac.jp

Ling Zhou

College of Applied Sciences, Xinjiang Agricultural University, Xinjiang,

Urumqi 830052, China

e-mail: zhoulin57030163.com

S. Li (Eds.): Nonlinear Maths for Uncertainty and its Appli., AISC 100, pp. 77-83. springerlink.com

(C) Springer-Verlag Berlin Heidelberg 2011 
In this note, we further discuss the relationship between convergence in measure and convergence pseudo-in measure for sequence of measurable functions. We shall introduce the concepts of strong autocontinuity from below and strong converse autocontinuity from below of a set function. By using the two types of autocontinuity from below of monotone measures, we investigate the inheriting of convergence in measure and convergence pseudo-in measure for sequence of measurable function under the common addition operation "+" and logic addition operation " $\vee$ ". The implication relationship between convergence in measure and pseudo-convergence in measure are shown by using autocontinuity from below and converse autocontinuity from below, respectively.

\section{Preliminaries}

Let $X$ be a non-empty set, $\mathcal{F}$ a $\sigma$-algebra of subsets of $X$, and $(X, \mathcal{F})$ denotes the measurable space.

Definition 1. ( 9, 15]) Set function $\mu: \mathcal{F} \rightarrow[0,+\infty]$ is called a monotone measure on $(X, \mathcal{F})$ iff it satisfies the following requirements:

(1) $\mu(\emptyset)=0$;

(vanishing at $\emptyset$ )

(2) $A \subset B$ and $A, B \in \mathcal{F} \Rightarrow \mu(A) \leq \mu(B)$.

(monotonicity)

When $\mu$ is a monotone measure, the triple $(X, \mathcal{F}, \mu)$ is called a monotone measure space ( 9,15$]$ ).

In some literature, a set function $\mu$ satisfying the conditions (1) and (2) of Definition 1 is called a fuzzy measure or a non-additive measure .

In this paper, all the considered sets are supposed to belong to $\mathcal{F}$ and $\mu$ is supposed to be a finite monotone measure, i.e., $\mu(X)<\infty$. All concepts and symbols not defined may be found in [9, 15].

Definition 2. ( 1]) A set function $\mu: \mathcal{F} \rightarrow[0,+\infty)$ is said to have pseudometric generating property (for short p.g.p), if for any $\left\{E_{n}\right\} \subset \mathcal{F}$ and $\left\{F_{n}\right\} \subset \mathcal{F}$

$$
\mu\left(E_{n}\right) \vee \mu\left(F_{n}\right) \rightarrow 0 \Longrightarrow \mu\left(E_{n} \cup F_{n}\right) \rightarrow 0
$$

Note: The concept of pseudometric generated property goes back to Dobrakov and Farkova in seventies, and this was related to Frechet-Nikodym topology [1,9].

Let $\mathbf{F}$ be the class of all finite real-valued measurable functions on $(X, \mathcal{F}, \mu)$, and let $A \in \mathcal{F}, f \in \mathbf{F}, f_{n} \in \mathbf{F}(n=1,2, \ldots)$ and $\left\{f_{n}\right\}$ denote a sequence of measurable functions. We say that $\left\{f_{n}\right\}$ converges in measure $\mu$ to 
$f$ on $A$, and denote it by $f_{n} \underset{A}{\stackrel{\mu}{\longrightarrow}} f$, if for any given $\sigma>0, \lim _{n \rightarrow+\infty} \mu\left(\left\{\left|f_{n}-f\right| \geq\right.\right.$ $\sigma\} \cap A)=0 ;\left\{f_{n}\right\}$ converges pseudo-in measure $\mu$ to $f$ on $A$, and denote it by $f_{n} \underset{A}{\stackrel{p, \mu}{\longrightarrow}} f$, if for any given $\sigma>0, \lim _{n \rightarrow+\infty} \mu\left(\left\{\left|f_{n}-f\right|<\sigma\right\} \cap A\right)=\mu(A) ;\left\{f_{n}\right\}$ converges pseudo-in measure $\mu$ to $f$ in $A$, and denote it by $f_{n} \stackrel{p . \mu}{\longrightarrow} f$ in $A$, if $f_{n} \stackrel{p . \mu}{\longrightarrow} f$ on $C$ for all $C \in A \cap \mathcal{F}$.

\section{Autocontinuity of Set Function}

In 14] Wang introduced the concepts of autocontinuity from below and converse-autocontinuity from below of set function, and discussed the convergence for sequence of measurable functions by using the structure of set functions. Now we shall introduce the concepts of strong autocontinuity from below and strong converse-autocontinuity from below for set functions and show their properties.

Definition 3. ( $9,14,15])$ Let $(X, \mathcal{F}, \mu)$ be a monotone measure space.

(1) $\mu$ is said to be autocontinuous from below and denote it by autoc. $\uparrow$, if for any $E \in \mathcal{F},\left\{F_{n}\right\} \subset \mathcal{F}$,

$$
\mu\left(F_{n}\right) \rightarrow 0 \Longrightarrow \mu\left(E-F_{n}\right) \rightarrow \mu(E) ;
$$

(2) $\mu$ is said to be converse-autocontinuous from below and denote it by c.autoc. $\uparrow$, if for any $A \in \mathcal{F},\left\{B_{n}\right\} \subset A \cap \mathcal{F}$,

$$
\mu\left(B_{n}\right) \rightarrow \mu(A) \Longrightarrow \mu\left(A-B_{n}\right) \rightarrow 0 .
$$

Definition 4. Let $(X, \mathcal{F}, \mu)$ be a monotone measure space.

(1) $\mu$ is said to be strong autocontinuous from below and denote it by s.autoc. $\uparrow$, if

$$
\mu\left(E_{n}\right) \vee \mu\left(F_{n}\right) \rightarrow 0 \Longrightarrow \mu\left(A-E_{n} \cup F_{n}\right) \rightarrow \mu(A),
$$

for any $A \in \mathcal{F},\left\{E_{n}\right\} \subset \mathcal{F}$ and $\left\{F_{n}\right\} \subset \mathcal{F}$;

(2) $\mu$ is said to be strong converse-autocontinuous from below and denote it by s.c.autoc. $\uparrow$, if

$$
\mu\left(A-E_{n}\right) \wedge \mu\left(A-F_{n}\right) \rightarrow \mu(A) \Longrightarrow \mu\left(E_{n} \cup F_{n}\right) \rightarrow 0,
$$

for any $A \in \mathcal{F},\left\{E_{n}\right\} \subset A \cap \mathcal{F}$ and $\left\{F_{n}\right\} \subset A \cap \mathcal{F}$.

Proposition 1. If $\mu$ is s.autoc. $\uparrow$ (resp. s.c.autoc. $\uparrow$ ), then it is autoc. $\uparrow$ (resp. c.autoc. $\uparrow)$.

Proposition 2. If $\mu$ is autoc. $\uparrow$ and has p.g.p, then it is s.autoc. $\uparrow$. 
Proposition 3. If $\mu$ is c.autoc. $\uparrow$ and has p.g.p, then it is s.c.autoc. $\uparrow$.

\section{Convergence in Measure}

In this section, we study the application relationship between convergence in measure and convergence pseudo-in measure on monotone measure spaces.

The first conclusion of the following theorem due to Wang [15].

Theorem 1. Let $\mu$ be a monotone measure. Then,

(1) $\mu$ is autoc. $\uparrow$ iff $f_{n} \underset{A}{\stackrel{p . \mu}{\longrightarrow}} f$ whenever $f_{n} \underset{A}{\longrightarrow} f, \forall A \in \mathcal{F}, f, f_{n} \in \mathbf{F}$;

(2) $\mu$ is c.autoc $\uparrow$, iff $f_{n} \underset{A}{\stackrel{\mu}{\longrightarrow}} f$ whenever $f_{n} \underset{A}{\stackrel{p . \mu}{\longrightarrow}} f, \forall A \in \mathcal{F}, f, f_{n} \in \mathbf{F}$.

Proof. We only prove (2). Let $\mu$ be c.autoco $\uparrow$. If $f_{n} \underset{A}{\stackrel{p . \mu}{\longrightarrow}} f$, then for any given $\sigma>0$, we have

$$
\lim _{n \rightarrow+\infty} \mu\left(\left\{\left|f_{n}-f\right|<\sigma\right\} \cap A\right)=\mu(A)
$$

and therefore, using the converse-autocontinuity from below of $\mu$, we have

$$
\begin{aligned}
\lim _{n \rightarrow+\infty} \mu\left(\left\{\left|f_{n}-f\right| \geq \sigma\right\} \cap A\right) & =\lim _{n \rightarrow+\infty} \mu\left(A-\left\{\left|f_{n}-f\right|<\sigma\right\}\right) \\
& =0 .
\end{aligned}
$$

So $f_{n} \underset{A}{\stackrel{\mu}{\longrightarrow}} f$.

Conversly, for any $A \in \mathcal{F},\left\{B_{n}\right\} \subset A \cap \mathcal{F}$, and $\mu\left(B_{n}\right) \rightarrow \mu(A)$, we define measurable function sequences $\left\{f_{n}\right\}$ by

$$
f_{n}=\chi_{B_{n}}=\left\{\begin{array}{lll}
0 & \text { if } & x \notin B_{n} \\
1 & \text { if } & x \in B_{n}
\end{array}\right.
$$

$\mathrm{n}=1,2, \ldots$, and denote $f \equiv 1$. It is easy to see that $f_{n} \underset{A}{\stackrel{p \cdot \mu}{\longrightarrow}} f$. If it implies $f_{n} \underset{A}{\stackrel{\mu}{\longrightarrow}} f$, then for $\sigma=\frac{1}{2}$, we have

$$
\lim _{n \rightarrow+\infty} \mu\left(\left\{\left|f_{n}-f\right| \geq \frac{1}{2}\right\} \cap A\right)=0
$$

As

$$
\left\{\left|f_{n}-f\right| \geq \frac{1}{2}\right\} \cap A=\left\{1-\chi_{B_{n}} \geq \frac{1}{2}\right\} \cap A=A-B_{n} .
$$

So $\lim _{n \rightarrow+\infty} \mu\left(A-B_{n}\right)=0$. This shows that $\mu$ is c.autoc $\uparrow$. 
The following theorems describe the inheriting of convergence in measure and convergence pseudo-in measure for sequence of measurable function under the common addition operation.

Theorem 2. Let $\mu$ be a monotone measure.

(1) If $\mu$ is s.autoc. $\uparrow$, then $f_{n} \stackrel{\mu}{\longrightarrow} f$ and $g_{n} \stackrel{\mu}{\longrightarrow} g$ on $A$ imply

$$
\alpha f_{n}+\beta g_{n} \underset{D}{\stackrel{p \cdot \mu}{D}} \alpha f+\beta g
$$

for any $D \in A \cap \mathcal{F}, \alpha, \beta \in R^{1}$.

(2) If $\mu$ is s.c.autoc. $\uparrow$, then $f_{n} \underset{A}{\stackrel{p . \mu}{\rightarrow}} f$ and $g_{n} \underset{A}{\stackrel{p . \mu}{\rightarrow}} g$ imply

$$
\alpha f_{n}+\beta g_{n} \underset{A}{\stackrel{\mu}{\longrightarrow}} \alpha f+\beta g
$$

for any $A \in \mathcal{F}, \alpha, \beta \in R^{1}$.

Proof. It is similar to the proof of Theorem 1

The following Theorem 3 and 4 describe respectively the characteristics of strong autocontinuity from below and strong converse-autocontinuity from below of set functions.

Theorem 3. The following statements are equivalent:

(1) $\mu$ is s.autoc. $\uparrow$;

(2) $f_{n}+g_{n} \underset{A}{\stackrel{p \cdot \mu}{\longrightarrow}} 0$ whenever $f_{n} \underset{A}{\stackrel{\mu}{\longrightarrow}} 0$ and $g_{n} \underset{A}{\stackrel{\mu}{\longrightarrow}} 0, \forall A \in \mathcal{F}$;

(3) $f_{n} \vee g_{n} \underset{A}{\stackrel{p \cdot \mu}{\longrightarrow}} 0$ whenever $f_{n} \underset{A}{\stackrel{\mu}{\longrightarrow}} 0$ and $g_{n} \underset{A}{\stackrel{\mu}{\longrightarrow}} 0, \forall A \in \mathcal{F}$.

Proof. (1) $\Longrightarrow(2)$. It follows directly from Theorem 2 above.

$(2) \Longrightarrow(3)$. For any $A \in \mathcal{F}$, if $f_{n} \underset{A}{\stackrel{\mu}{\longrightarrow}} 0$ and $g_{n} \underset{A}{\stackrel{\mu}{\longrightarrow}} 0$, then $\left|f_{n}\right| \stackrel{\mu}{A} 0$ and $\left|g_{n}\right| \stackrel{\mu}{\rightarrow} 0$. By condition (2), we have $\left|f_{n}\right|+\left|g_{n}\right| \stackrel{p \cdot \mu}{\longrightarrow} 0$ on $A$, therefore, for any $\sigma>0$,

$$
\lim _{n \rightarrow+\infty} \mu\left(\left\{\left|f_{n}\right|+\left|g_{n}\right|<\sigma\right\} \cap A\right)=\mu(A) .
$$

Noting that $\left|f_{n} \vee g_{n}\right| \leq\left|f_{n}\right|+\left|g_{n}\right|$, we get

$$
\left\{\left|f_{n}\right|+\left|g_{n}\right|<\sigma\right\} \cap A \subseteq\left\{\left|f_{n} \vee g_{n}\right|<\sigma\right\} \cap A \subseteq A .
$$

So

$$
\lim _{n \rightarrow+\infty} \mu\left(\left\{\left|f_{n} \vee g_{n}\right|<\sigma\right\} \cap A\right)=\mu(A) .
$$

This shows $f_{n} \vee g_{n} \stackrel{p . \mu}{\longrightarrow} 0$ on $A$. 
$(3) \Longrightarrow(1)$. For any $\left\{E_{n}\right\} \subset \mathcal{F},\left\{F_{n}\right\} \subset \mathcal{F}$ with $\lim _{n \rightarrow \infty} \mu\left(E_{n}\right) \vee \mu\left(F_{n}\right)=0$, we define measurable function sequences $\left\{f_{n}\right\}$ and $\left\{g_{n}\right\}$ by

$$
f_{n}=\chi_{E_{n}}=\left\{\begin{array}{l}
0 \text { if } x \notin E_{n} \\
1 \text { if } x \in E_{n}
\end{array}\right.
$$

and

$$
g_{n}=\chi_{F_{n}}=\left\{\begin{array}{lll}
0 & \text { if } & x \notin F_{n} \\
1 & \text { if } & x \in F_{n}
\end{array}\right.
$$

$\mathrm{n}=1,2, \ldots$, then $f_{n} \stackrel{\mu}{\longrightarrow} 0$ on $A$ and $g_{n} \stackrel{\mu}{\longrightarrow} 0$ on $A$. Thus, $f_{n} \vee g_{n} \stackrel{\mu}{\longrightarrow} 0$ on $A$. Therefore for $\sigma=\frac{1}{2}$, we have

$$
\lim _{n \rightarrow+\infty} \mu\left(\left\{f_{n} \vee g_{n}<\frac{1}{2}\right\} \cap A\right)=\mu(A) \text {. }
$$

Noting $f_{n} \vee g_{n}=\chi_{E_{n}} \vee \chi_{F_{n}}=\chi_{E_{n} \cup F_{n}}$, and

$$
\left\{\chi_{E_{n}} \vee \chi_{F_{n}}<\frac{1}{2}\right\} \cap A=A-\left\{\chi_{E_{n}} \vee \chi_{F_{n}} \geq \frac{1}{2}\right\}=A-E_{n} \cup F_{n}
$$

So

$$
\lim _{n \rightarrow+\infty} \mu\left(A-E_{n} \cup F_{n}\right)=\mu(A) .
$$

That is, $\mu$ is s.autoc. $\uparrow$.

Theorem 4. The following statements are equivalent:

(1) $\mu$ is s.c.autoc. $\uparrow$;

(2) $f_{n}+g_{n} \underset{A}{\stackrel{\mu}{\longrightarrow}} 0$ whenever $f_{n} \underset{A}{\stackrel{p \cdot \mu}{\longrightarrow}} 0$ and $g_{n} \underset{A}{\stackrel{p . \mu}{\longrightarrow}} 0, \forall A \in \mathcal{F}$;

(3) $f_{n} \vee g_{n} \underset{A}{\stackrel{\mu}{\longrightarrow}} 0$ whenever $f_{n} \underset{A}{\stackrel{p \cdot \mu}{\longrightarrow}} 0$ and $g_{n} \underset{A}{\stackrel{p \cdot \mu}{\longrightarrow}} 0, \forall A \in \mathcal{F}$.

Proof. It is similar to the proof of Theorem 3 .

Acknowledgements. The first author was supported by NSFC Grant No. 70771010. The second author was supported by JSPS Grant No.22540112.

\section{References}

1. Dobrakov, I., Farkova, J.: On submeasures II. Math. Slovoca 30, 65-81 (1980)

2. Li, J.: Order continuous of monotone set function and convergence of measurable functions sequence. Applied Mathematics and Computation 135(2-3), 211-218 (2003)

3. Liu, Y., Liu, B.: The relationship between structural characteristics of fuzzy measure and convergences of sequences of measurable functions. Fuzzy Sets and Systems 120, 511-516 (2001) 
4. Li, J., Mesiar, R., Zhang, Q.: Absolute continuity of monotone measure and convergence in measure. Communications in Computer and Information Science 80, 500-504 (2010)

5. Li, J., Yasuda, M., Jiang, Q., Suzuki, H., Wang, Z., Klir, G.J.: Convergence of sequence of measurable functions on fuzzy measure space. Fuzzy Sets and Systems 87, 385-387 (1997)

6. Li, J., Yasuda, M.: On Egoroff's theorem on finite monotone non-additive measure space. Fuzzy Sets and Systems 153, 71-78 (2005)

7. Li, J., Zhang, Q.: Asymptotic structural characteristics of monotone measure and convergence in monotone measure. The Journal of Fuzzy Mathematics 9(2), 447-459 (2001)

8. Murofushi, T., Uchino, K., Asahina, S.: Conditions for Egoroff's theorem in non-additive measure theory. Fuzzy Sets and Systems 146, 135-146 (2004)

9. Pap, E.: Null-additive Set Functions. Kluwer Academic Press, Dordrecht (1995)

10. Song, J., Li, J.: Lebesgue theorems in non-additive measure theory. Fuzzy Sets and Systems 149, 543-548 (2005)

11. Sun, Q.: Property $(S)$ of fuzzy measure and Riesz's theorem. Fuzzy Sets and Systems 62, 117-119 (1994)

12. Takahashi, M., Murofushi, T.: Relationship between convergence concepts in fuzzy measure theory. In: Proc. 11th IFSA World Congress, vol. I, pp. 467-473 (2005)

13. Uchino, K., Murofushi, T.: Relations between mathematical properties of fuzzy measures. In: Proc. 10th IFSA World Congress, pp. 27-30 (2003)

14. Wang, Z.: Asymptotic structural characteristics of fuzzy measure and their applications. Fuzzy Sets and Systems 16, 277-290 (1985)

15. Wang, Z., Klir, G.J.: Generalized Measure Theory. Springer, Boston (2009) 